\title{
VITAMIN D DEFICIENCY IN ACUTE CORONARY SYNDROME - CLINICALLY RELEVANT OR INCIDENTAL FINDING?
}

\author{
Marijana Knežević Praveček ${ }^{1}$, Željka Vuković-Arar², Blaženka Miškić ${ }^{3}$, Irzal Hadžibegović ${ }^{1}$ \\ ${ }^{1}$ Department of Cardiology, Dr. Josip Benčević General Hospital, School of Medicine, Josip Juraj Strossmayer University, Osijek, Croatia \\ ${ }^{2}$ Department of Ophthalmology, Dr. Josip Benčević General Hospital, School of Medicine, Josip Juraj Strossmayer University, Osijek, Croatia \\ ${ }^{3}$ Department of Endocrinology, Dr. Josip Benčević General Hospital, School of Medicine, Josip Juraj Strossmayer University, Osijek, Croatia
}

\section{SUMMARY}

Objective: Vitamin D deficiency has been associated with cardiovascular disease. The aim of this study was to determine serum concentration of 25 hydroxyvitamin $\mathrm{D}(25(\mathrm{OH}) \mathrm{D})$ in patients with acute coronary syndrome (ACS) and to assess the prognostic role of serum vitamin D level in ACS patients during 3-year follow up.

Methods: The study included 60 ACS patients hospitalized at cardiology department for ACS between March 2012 and October 2012, and 60 age- and sex-matched control patients without ACS. Standard laboratory testing and vitamin D determination were performed in all study patients. In addition, ACS patients underwent coronarography and were followed-up for 36 months of ACS for major adverse cardiac events (MACE).

Results: Patients with ACS had a statistically significantly lower mean $25(\mathrm{OH}) \mathrm{D}$ level as compared with control group ( $35.19 \mathrm{nmol} / \mathrm{L}$ vs. 58.08 $\mathrm{nmol} / \mathrm{L}, \mathrm{p}<0.001)$. The lowest mean level of $25(\mathrm{OH}) \mathrm{D}$ was recorded in diabetic patients with ACS (30.45 nmol/L). ACS patients were divided into three subgroups according to coronarography findings: single vessel, double vessel and triple vessel coronary artery disease (CAD) with respective serum levels of $25(\mathrm{OH}) \mathrm{D}$ of $36.44 \mathrm{nmol} / \mathrm{L}, 33.65 \mathrm{nmol} / \mathrm{L}$ and $31.70 \mathrm{nmol} / \mathrm{L}$. During 36 -month follow up, the event-free survival rate was $60 \%$ in the ACS group. The ACS patients having sustained MACE during follow up had low serum level of 25(OH)D in the acute phase; however, the difference from ACS patients without MACE during follow up did not reach statistical significance (32.64 nmol/L vs. $37.01 \mathrm{nmol} / \mathrm{L})$.

Conclusions: Patients with ACS have low vitamin D level, which is lowest in diabetic patients with ACS. However, during 3-year follow up, vitamin $\mathrm{D}$ failed to prove useful as a prognostic biomarker in ACS patients.

Key words: acute coronary syndrome, coronary angiography, 25-hydroxyvitamin D, diabetes, vitamin D deficiency

Address for correspondence: M. Knežević Praveček, Department of Cardiology, Dr. Josip Benčević General Hospital, Andrije Štampara 42, HR-35000 Slavonski Brod, Croatia. E-mail: marijana@aip.hr

https://doi.org/10.21101/cejph.a4577

\section{INTRODUCTION}

Vitamin D is a precursor of the steroid hormone calcitriol which is necessary for mineral homeostasis and bone metabolism (1). Upon vitamin D discovery in almost all body cells, such as immune system, vascular and myocardial cells, a more diversified role of vitamin D than previously believed has been recognized. This has resulted in extensive research into the role of vitamin $\mathrm{D}$ in the pathogenesis of chronic non-skeletal diseases such as infectious, autoimmune, malignant, and cardiovascular diseases (CVD) (2).

Cardiovascular risk factors such as arterial hypertension, obesity, dyslipidemia, and diabetes mellitus (DM), and the resulting CVD including acute coronary syndrome (ACS), coronary artery disease (CAD) and stroke are the most prevalent illnesses and the leading causes of death worldwide. Therefore, it is of utmost importance to determine the role of vitamin D in CVD.

Vitamin D deficiency has been recorded all over the world. Estimates of vitamin D status in various European countries show great differences. According to literature data, the prevalence of vitamin D deficiency is between 2\% and 30\% in adult European population; however, some studies report a figure as high as $75 \%$ in elderly institutionalized individuals. Accordingly, vitamin D deficiency has grown into a major public health problem (3).

The 1,25(OH)2D is a bioactive form of vitamin $\mathrm{D}$, while 25 hydroxyvitamin D (25(OH)D) is the best indicator of vitamin D status. Serum concentration of 25(OH)D reflects the level of vitamin D synthesized in the dermis as a function UV radiation and dietary vitamin D intake. Cold season, old age, female sex, obesity, dark skin, inadequate sun exposure, fat malabsorption, and poor dietary habits along with the absence of dietary vitamin $\mathrm{D}$ enrichment are the major risk factors for vitamin D deficiency.

In scientific community, there is no consensus on the adequate vitamin D level. The opinions concerning optimal and borderline 25(OH)D levels vary considerably. According to the Institute of Medicine Committee to Review Dietary Reference Intakes for Vitamin D and Calcium, serum 25(OH)D level $\geq 50 \mathrm{nmol} / \mathrm{L}$ is considered normal, $30-50 \mathrm{nmol} / \mathrm{L}$ inadequate and $\leq 30 \mathrm{nmol} / \mathrm{L}$ deficient. The recommended level of $50 \mathrm{nmol} / \mathrm{L}$ is based on evidence on the vitamin D level necessary for bone health. On 
the other hand, according to the recommendations issued by the American Geriatrics Society, International Osteoporosis Foundation and American Association of Clinical Endocrinologists, serum $25(\mathrm{OH}) \mathrm{D}$ level $<50 \mathrm{nmol} / \mathrm{L}$ is considered inadequate and $>75 \mathrm{nmol} / \mathrm{L}$ appropriate to reduce the risk of fractures and falls in the elderly $(4,5)$. There is ever more evidence pointing to a U-shaped curve for several vitamin D level associated health outcomes, including CVD and all-cause mortality, the risk being lowest at moderate but increased at low and high $25(\mathrm{OH})$ D levels (6).

Vitamin D deficiency was found in patients with ACS and preliminary studies suggest it to be potentially associated with prognosis as well. However, convincing evidence confirming the impact of vitamin D insufficiency or deficiency on ACS patient morbidity and mortality is still lacking (7). The biological mechanism of vitamin D deficiency in ACS is not simple; we can only speculate on a dual effect of vitamin D in ACS, i.e. its direct immunomodulatory action and indirect action on the known cardiovascular risk factors.

There is evidence for vitamin D to suppress the renin-angiotensin system and to influence endothelial function, inflammatory processes, platelet function, insulin resistance, and blood pressure (8). All these effects are highly relevant in ACS influencing the patient clinical course. In addition, low vitamin D level has been associated with ventricular dysfunction and cardiac remodeling following ACS, as well as with heart failure and sudden cardiac death (9-12). Therefore, it appears that vitamin D level can influence both short-term and long-term outcomes in ACS patients.

The aim of the present study was to analyze serum 25(OH)D levels in ACS patients in comparison to ACS-free control group, to assess the association of CAD and serum 25(OH)D levels in ACS patients, and to evaluate the ACS patient prognosis according to serum 25(OH)D levels for the first time in a homogeneous Caucasian population in Croatia.

\section{MATERIALS AND METHODS}

\section{Study Population}

The study included 60 patients treated for ACS at Dr Josip Benčević General Hospital in Slavonski Brod, Croatia, from March 2012 till October 2012, and 60 subjects not treated for any form of ischaemic heart disease before enrolment in the study as a control group. We included patients with ST elevation myocardial infarction (STEMI) and those with non-ST elevation acute coronary syndrome (NSTE-ACS). The inclusion criteria for ACS group were age (30-70 years); guideline confirmed acute $\leq 12$-hour STEMI and acute $\leq 24$-hour NSTE-ACS; and scheduled coronarography with revascularization (urgent for STEMI and early within 24 hours for NSTE-ACS) $(13,14)$.

The ACS patients were divided into three subgroups according to coronarography findings: single vessel coronary artery disease (SVCAD) group, double vessel coronary disease (DVCAD) group, and multivessel coronary disease group with three or more coronary arteries involved, including the left coronary artery main trunk (MVCAD).

Control group consisted of 60 age- and sex-matched subjects free from ACS but with at least one of the established risk factors for CAD, including hypertension, DM, obesity, smoking, and hyperlipidemia. Individuals with known recent or previous ACS, or established CVD or chronic disease such as renal or hepatic disease, hyperthyroidism or hyperparathyroidism, sarcoidosis, tuberculosis, autoimmune disease, malabsorption, bone disease, malignancy, pregnancy or lactation, immobility for more than one week, recent vitamin D administration (oral in the last two weeks or injection in the last two months), and receiving drugs affecting vitamin D and calcium metabolism (e.g. corticosteroids or antiepileptic drugs) were excluded. In order to minimize the confounding effect of seasonal fluctuation of 25(OH)D concentration, we tended to involve an equal number of patients each month.

Blood samples were obtained on patient admission. Creatinine, total cholesterol, high-density lipoprotein (HDL) cholesterol, low-density lipoprotein (LDL) cholesterol, triglycerides, glucose, and total calcium were determined colorimetrically on an AU 680 (Beckman Coulter, Fullerton, USA) auto-analyzer using respective reagents of the same manufacturer. The following demographic and medical data were recorded: age, gender, body mass index (body weight in kilograms divided by the square of height in metres, $\mathrm{kg} / \mathrm{m}^{2}$, BMI), smoking habit, DM, and hypertension. Smoking habit was classified into two categories: current/ ex-smoker and non-smoker. Diabetes mellitus was classified as newly detected DM (fasting blood glucose $>7 \mathrm{mmol} / \mathrm{L}$ ) and known DM treated with insulin or oral hypoglycemic agents. Arterial hypertension included newly detected hypertension with diastolic blood pressure $>90 \mathrm{~mm} \mathrm{Hg}$ ( $>85 \mathrm{~mm} \mathrm{Hg}$ in diabetic patients) or systolic blood pressure $>145 \mathrm{~mm} \mathrm{Hg}$ (>130 mm $\mathrm{Hg}$ in diabetic patients) and known hypertension treated with antihypertensive therapy.

Plasma parathyroid hormone (PTH) and vitamin D were determined by the electrochemiluminescence method on an Elecsys 2010, Cobas e 411 (Roche Diagnostics, Mannheim, Germany) analyzer with respective reagents. The Institute of Medicine Food and Nutrition Board Dietary Reference Intakes for Vitamin D classifies $25(\mathrm{OH}) \mathrm{D}$ serum concentration $\geq 50 \mathrm{nmol} / \mathrm{L}(\geq 20 \mathrm{ng} /$ $\mathrm{mL})$ as normal, $30-50 \mathrm{nmol} / \mathrm{L}(12-20 \mathrm{ng} / \mathrm{mL})$ as insufficient, and $\leq 30 \mathrm{nmol} / \mathrm{L}(\leq 12 \mathrm{ng} / \mathrm{mL})$ as deficient (15).

The study was approved by the Ethics Committee of the Osijek School of Medicine, Josip Juraj Strossmayer University, Osijek. All patients provided their written informed consent.

\section{Prognostic Parameters}

Prognosis of ACS patients was assessed according to survival rate for the following MACE: death, reinfarction, target lesion revascularization, cardiac rehospitalization, and stroke during 36-month follow up. Data were collected during medical examination, from medical records and by telephone interview with the patient or patient family member.

\section{Statistics}

Categorical variables were presented descriptively and tested by $\chi^{2}$-test for between-group differences. Shapiro-Wilk test was employed to test the normality of result distribution of all numerical continuous variables according to the number of patients per group. Of all the variables observed, only vitamin D value showed normal distribution across the sample and within particular groups, 
whereas all other variables failed to show normal distribution.

Numerical variables with normal distribution were presented with the mean and standard deviation (SD), while between-group differences were analyzed by Student's t-test. Differences in numerical variables with normal distribution among more than two groups were analyzed by ANOVA with post hoc analysis using Tukey HSD post hoc test.

Numerical variables with non-normal distribution were presented with median value and minimal to maximal value range. Between-group differences in numerical variables with nonnormal distribution were analyzed by Mann-Whitney test for two independent samples.

Correlations between continuous numerical variables with normal distribution were tested by Pearson correlation test, whereas the variables with non-normal distribution were tested by Spearman correlation test. Correlation of the study variables with vitamin $\mathrm{D}$ values within the groups was analyzed by linear regression.

The level of statistical significance was set at $\mathrm{p}<0.05$. Statistical analysis was performed by use of the SPSS for Windows 11.0.3 software (SPSS Inc., Chicago, IL, USA).

\section{RESULTS}

The study included 60 ACS patients and 60 patients without ACS as a control group. There were no significant differences in demographic and relevant clinical characteristics between the two groups of patients, except for a statistically significantly higher proportion of smokers in the ACS group as compared with the control group (Table 1).

The ACS group included 36 (60\%) patients with STEMI and 24 (40\%) patients with NSTE-ACS. According to the coronary disease severity assessed by coronarography, patients were divided into subgroups with SVCAD - 39 (68\%), DVCAD - 15 (26\%) and MVCAD - 3 (6\%).

The mean serum concentration of 25(OH)D of 35.19 \pm 17.54 $\mathrm{nmol} / \mathrm{L}$ measured in ACS patients was statistically significantly lower in comparison with control group, where it was $58.08 \pm 16.29 \mathrm{nmol} / \mathrm{L}$ (Student's t-test, $\mathrm{p}<0.001$ ) (Table 1).

Use of reference values for vitamin D sufficiency, insufficiency and deficiency yielded a statistically significantly higher rate of vitamin D insufficiency among ACS patients as compared with control group $\left(\chi^{2}\right.$-test, $\left.\mathrm{p}<0.001\right)$. Vitamin $\mathrm{D}$ deficiency was recorded in as many as $20 \%$ of ACS patients and none of control subjects (Fig. 1). There was no statistically significant difference in 25(OH)D serum levels within ACS and control groups according to age and other relevant clinical characteristics, except for DM (Table 1). According to gender, a lower mean 25(OH) $\mathrm{D}$ level was recorded in female ACS patients $(30.85 \mathrm{nmol} / \mathrm{L}$ vs. $37.35 \mathrm{nmol} / \mathrm{L})$, but not in control female patients, where a higher mean 25(OH)D level was measured $(61.84 \mathrm{nmol} / \mathrm{L}$ vs. 55.90 $\mathrm{nmol} / \mathrm{L})$; these differences did not reach statistical significance either (Student's t-test, $\mathrm{p}>0.05$ ).

In the ACS group, the mean 25(OH)D level was lower in patients with DM comorbidity. In addition, the lowest mean $25(\mathrm{OH}) \mathrm{D}$ level of $30.45 \pm 15.05 \mathrm{nmol} / \mathrm{L}$ was recorded in the subgroup of ACS + DM patients as compared with all other study groups. A similar tendency toward a lower mean 25(OH)D level
Table 1. Baseline characteristics of study subjects

\begin{tabular}{|l|c|c|}
\hline \multirow{2}{*}{ Characteristic } & \multicolumn{2}{|c|}{ Group of subjects, $\mathrm{N}(\%)$} \\
\cline { 2 - 3 } & $\begin{array}{c}\text { Acute } \\
\text { coronary } \\
\text { syndrome } \\
\mathrm{N}=60\end{array}$ & $\begin{array}{c}\text { Control } \\
\mathrm{N}=60\end{array}$ \\
\hline Gender, male, $\mathrm{n}(\%)$ & $40(67)$ & $38(63)$ \\
\hline Age, median (range) & $58(34-72)$ & $58.5(35-72)$ \\
\hline BMl, median (range) & $28(20-34)$ & $27(20-42)$ \\
\hline Diabetes mellitus, $\mathrm{n}(\%)$ & $21(35)$ & $15(25)$ \\
\hline Smoking, $\mathrm{n}(\%)$ & $39(67)$ & $28(47)^{\star}$ \\
\hline Hypertension, $\mathrm{n}(\%)$ & $43(72)$ & $33(55)$ \\
\hline Dyslipidemia, $\mathrm{n}(\%)$ & $52(87)$ & $44(73)$ \\
\hline Creatinine, mean $\pm \mathrm{SD}(\mu \mathrm{mol} / \mathrm{L})$ & $89.98 \pm 13.12$ & $81.73 \pm 12.22$ \\
\hline Total cholesterol, mean $\pm \mathrm{SD}(\mathrm{mmol} / \mathrm{L})$ & $5.45 \pm 1.21$ & $5.73 \pm 1.13$ \\
\hline Triglycerides, mean $\pm \mathrm{SD}(\mathrm{mmol} / \mathrm{L})$ & $2.10 \pm 1.25$ & $1.87 \pm 1.12$ \\
\hline LDL cholesterol, mean $\pm \mathrm{SD}(\mathrm{mmol} / \mathrm{L})$ & $3.22 \pm 1.16$ & $3.44 \pm 1.03$ \\
\hline HDL cholesterol, mean $\pm \mathrm{SD}(\mathrm{mmol} / \mathrm{L})$ & $1.00 \pm 0.30$ & $1.14 \pm 0.29$ \\
\hline Calcium, mean $\pm \mathrm{SD}(\mathrm{mmol} / \mathrm{L})$ & $2.29 \pm 0.14$ & $2.37 \pm 0.11$ \\
\hline Phosphorus, mean $\pm \mathrm{SD}(\mathrm{mmol} / \mathrm{L})$ & $1.10 \pm 0.19$ & $1.18 \pm 0.15$ \\
\hline PTH, mean $\pm \mathrm{SD}(\mathrm{pg} / \mathrm{mL})$ & $36.14 \pm 10.69$ & $31.79 \pm 9.12$ \\
\hline 25 OH vitamin $\mathrm{D}, \mathrm{mean} \pm \mathrm{SD}(\mathrm{nmol} / \mathrm{L})$ & $35.19 \pm 17.54$ & $58.08 \pm 16.29+$ \\
\hline SVCAD, $\mathrm{n}(\%)$ & $39(68)$ & $\mathrm{NA}$ \\
\hline DVCAD, $\mathrm{n}(\%)$ & $15(26)$ & $\mathrm{NA}$ \\
\hline MVCAD, $\mathrm{n}(\%)$ & $3(6)$ & $\mathrm{NA}$ \\
\hline PCl, $\mathrm{n}(\%)$ & $46(77)$ & $\mathrm{NA}$ \\
\hline CABG, $\mathrm{n}(\%)$ & $9(15)$ & $\mathrm{NA}$ \\
\hline OMT, $\mathrm{n}(\%)$ & $5(8)$ & $\mathrm{NA}$ \\
\hline Total MACE, $\mathrm{n}(\%)$ & $24(40)$ & $\mathrm{NA}$ \\
\hline
\end{tabular}

${ }^{*}$ chi square test, $p<0.05$

'Student's t-test, $p<0.001$

BMI - body mass index, LDL - low-density lipoprotein, HDL - high-density lipoprotein, PTH - parathyroid hormone, $250 \mathrm{H}$ vitamin D - 25-hydroxyvitamin D, SVCAD - single vessel coronary artery disease, DVCAD - double vessel coronary artery disease, MVCAD - multivessel coronary artery disease, $\mathrm{PCl}$ - percutaneous coronary intervention, CABG - coronary artery bypass graft, OMT - optimal medical therapy, MACE - major adverse cardiac events

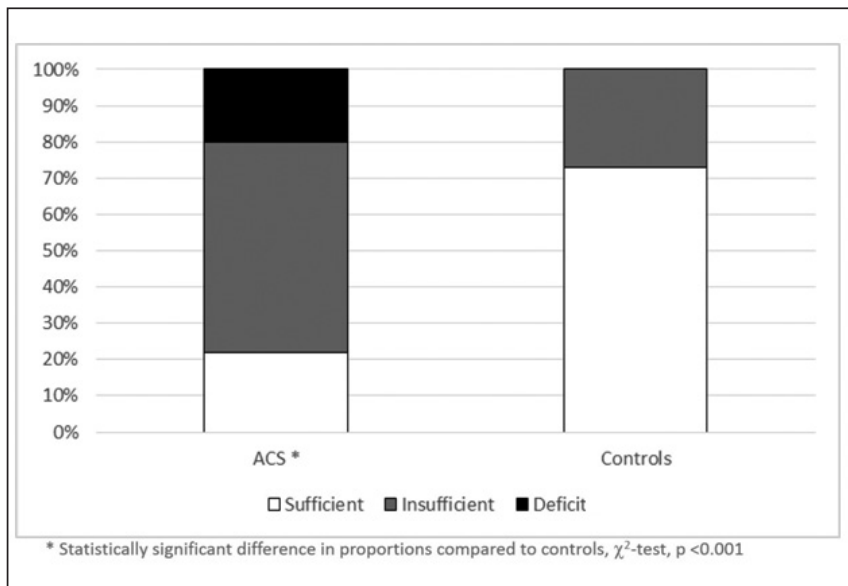

Fig. 1. Significant differences in the proportion of patients with 25(OH)D sufficiency, insufficiency and deficit between ACS and control groups of patients. 


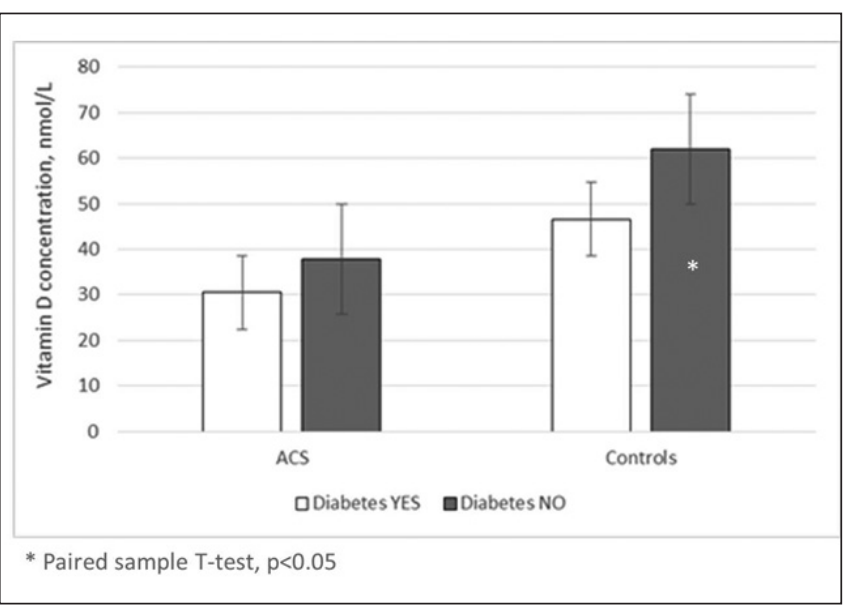

Fig. 2. Lower $25(\mathrm{OH}) \mathrm{D}$ concentration in patients with diabetes mellitus (DM) in both acute coronary syndrome (ACS) group and control group.

of $46.60 \pm 19.26 \mathrm{nmol} / \mathrm{L}$ in DM patients was also recorded in control group, and this difference was statistically significant (Student's t-test, $\mathrm{p}<0.05$ ) (Fig. 2). In the group of ACS patients, there was no difference in serum 25(OH)D level between STEMI and NSTE-ACS patients.

According to CAD severity, the lowest 25(OH)D mean level was measured in patients with MVCAD $(31.70 \mathrm{nmol} / \mathrm{L})$. The mean 25(OH)D levels were lower in patients with DVCAD as compared with those with SVCAD (33.65 nmol/L vs. 36.44 $\mathrm{nmol} / \mathrm{L}$ ), however, the difference was not statistically significant (one-way ANOVA, p=0.818; post hoc Tukey HSD test).

There were no significant correlations of 25(OH)D concentration with BMI, LDL-cholesterol, HDL-cholesterol and triglycerides, but they differed greatly between the ACS group and control group; $25(\mathrm{OH}) \mathrm{D}$ correlation with LDL-cholesterol and HDL-cholesterol was positive in ACS patients but negative in control patients. Also, correlation of 25(OH)D and triglycerides was negative in ACS patients but positive in control patients. However, none of these correlations was statistically significant. Positive correlation of 25(OH)D and LDL-cholesterol in ACS patients was close to statistical significance; it was submitted to linear regression analysis, which yielded a weak correlation of these two variables, based on the linear regression curve shape with a great number of outliers, with a low level of correlation $\left(\mathrm{R}^{2}=0.059\right)$.

During 36-month follow up, $40 \%$ of study patients suffered MACE. Serum level of 25(OH)D in acute phase was low in ACS patients with MACE, but difference from ACS patients without MACE was not statistically significant (32.64 nmol/L vs. $37.01 \mathrm{nmol} / \mathrm{L}$ ) (Fig. 3). The number of ACS patients with MACE recorded during follow up was comparable in all three patient subgroups with normal, insufficient and deficient 25(OH) D serum levels (Fig. 4).

\section{DISCUSSION}

The present study conducted in Croatia compared serum 25(OH)D levels in ACS patients and age-matched non-ACS patients as a control group. Serum 25(OH)D level was statistically

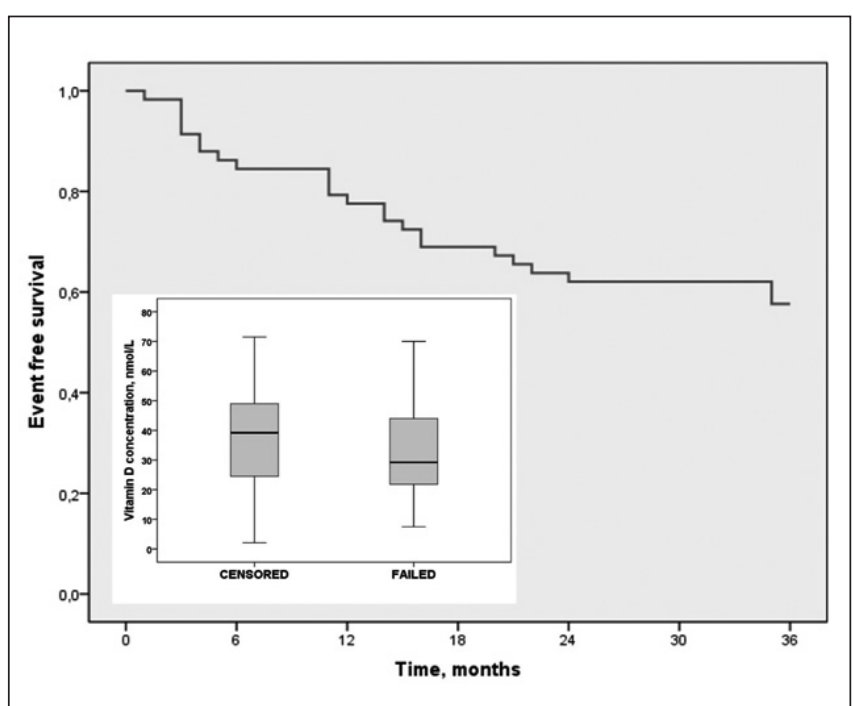

Fig. 3. Event-free survival in total ACS group and differences in $25(\mathrm{OH}) \mathrm{D}$ concentration according to adverse events.

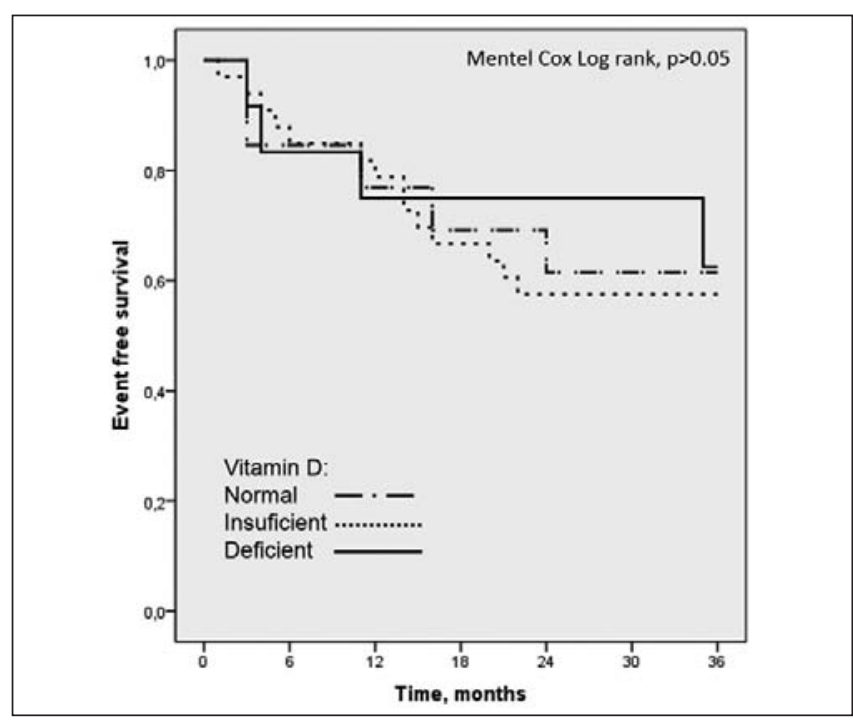

Fig. 4. Similar event-free survival in ACS patients with normal, insufficient or deficient 25(OH)D.

significantly lower in the former group of patients. Our results on the high prevalence of vitamin D deficiency or insufficiency in ACS patients (80\%) are consistent with literature data associating CVD and numerous CVD risk factors with 25(OH)D deficiency $(6,8-10,16)$. In addition to the high prevalence of $25(\mathrm{OH}) \mathrm{D}$ deficiency in ACS patients, our study confirmed association of demographic characteristics including female gender and DM with vitamin D deficiency (17).

It should be noted that the study included a homogeneous Caucasian population. Geographically, Croatia lies mostly between latitudes $42^{\circ}$ and $46^{\circ} \mathrm{N}$, thus preventing vitamin $\mathrm{D}$ production in the skin by sun exposure from October to March. Being aware of vitamin D seasonal variation, the study was conducted from March till October (18).

Study results confirmed significant association between low vitamin D level and higher prevalence of DVCAD and MVCAD 
in ACS. In their comparison study, Correia et al. demonstrated the association of vitamin D insufficiency and coronary artery atherosclerosis in stable coronary disease (19), while Verdoia et al. reported a significant association of vitamin D deficiency and severe CVD, in particular in patients with 25(OH)D level $<24.96 \mathrm{nmol} / \mathrm{L}(20)$.

The possible association of vitamin D deficiency and ACS should be additionally elucidated in future studies. The potential mechanisms by which vitamin D insufficiency might lead to an increased cardiovascular risk, directly or indirectly leading to hyperparathyroidism, may include activation of the reninangiotensin system and impaired insulin synthesis, secretion and sensitivity, thus influencing glycemic control and favouring development of DM (21). Low 25(OH)D levels have also been associated with an increased risk of macrovascular and microvascular complications in DM type 2 (22).

Other potential sequels of vitamin D deficiency include exacerbation of atherosclerosis, inflammation, and precipitation of arterial calcification. Vitamin D deficiency has been associated with endothelial dysfunction, which in turn is associated with an increased risk of cardiovascular events (23).

In our study, we demonstrated low serum vitamin D level in the acute phase of ACS; however, we failed to confirm the association of low serum vitamin D level and MACE in ACS patients during 3-year follow up. In recent years, there has been even greater interest in the possible sequels of low vitamin D level in ACS. De Metrio et al. reported the possible correlation between low vitamin D level and increased in-hospital mortality; however, due to the small sample size, they found no difference in mortality between ACS patients with normal and low vitamin D levels (24). In another study, the authors pointed to the possible independent association of vitamin D deficiency and in-hospital cardiovascular mortality (19). The largest study assessing vitamin D level and prognosis in ACS patients, conducted by Ng et al., found the lowest vitamin D concentration to be associated with long-term nonfatal MACE in ACS patients (11).

Our study has some limitations, i.e. only patients hospitalized at a single centre were included. Small study sample reduces the power of event-free survival analysis and the results obtained thus cannot be extrapolated to the overall population of ACS patients. Therefore, these data can only be used to generate some hypotheses, since they offer no evidence supporting the causative relationship; this would require confirmation in an appropriately randomized clinical trial.

In conclusion, vitamin D deficiency is present in the majority of ACS patients, with the lowest vitamin D level measured in diabetic ACS patients. During 3-year follow up, vitamin D failed to prove useful as a prognostic biomarker in ACS patients.

However, the results obtained may prove relevant for public health by pointing to the issue, considering the high prevalence of vitamin D deficiency, aging of the population, polypragmasia contributing to low vitamin D levels, and sedentary lifestyle (25). Vitamin D deficiency can be treated and vitamin D supplementation is inexpensive; however, we should wait for the results of large randomized interventional studies that will determine whether CVD morbidity and mortality are reduced by vitamin D supplementation.

\section{REFERENCES}

1. Bikle DD. Vitamin D metabolism, mechanism of action, and clinical applications. Chem Biol. 2014;21(3):319-29.

2. Ryan JW, Anderson PH, Morris HA. Pleiotropic activities of vitamin D receptors - adequate activation for multiple health outcomes. Clin Biochem Rev. 2015;36(2):53-61.

3. Hilger J, Friedel A, Herr R, Rausch T, Roos F, Wahl DA, et al. A systematic review of vitamin D status in populations worldwide. Br J Nutr. 2014;111:23-45.

4. Zittermann A, Prokop S. The role of vitamin D for cardiovascular disease and overall mortality. Adv Exp Med Biol. 2014;810:106-19.

5. Ross CA, Taylor CL, Yaktine AL, Del Valle HB, editors. Dietary reference intakes for calcium and vitamin D. Washington, DC: The National Academies Press, 2011.

6. Naesgaard PA, León de la Fuente RA, Nilsen ST, Pönitz V, BrüggerAndersen T, Grundt H, et al. Suggested cut-off values for vitamin D as a risk marker for total and cardiac death in patients with suspected acute coronary syndrome. Front Cardiovasc Med. 2016;3:4. doi: 10.3389/ fcrm.2016.00004.

7. Eren E, Ellidag HY, Yılmaz A, Aydın Ö, Yılmaz N. No association between vitamin $\mathrm{D}$ levels and inflammation markers in patients with acute coronary syndrome. Adv Med Sci. 2015;60(1):89-93.

8. Santoro D, Caccamo D, Lucisano S, Buemi M, Sebekova K, Teta D, et al. Interplay of vitamin D, erythropoiesis, and the renin-angiotensin system. BioMed Res Int. 2015;2015:145828. doi: 10.1155/2015/145828.

9. Karakas M, Thorand B, Zierer A, Huth C, Meisinger C, Roden M, et al. Low levels of serum 25-hydroxyvitamin $\mathrm{D}$ are associated with increased risk of myocardial infarction, especially in women: results from the MONICA/KORAAugsburg case-cohort study. J Clin Endocrinol Metab. 201;98(1):272-80.

10. Mahdavi K, Amirajam Z, Yazdankhah S, Majidi S, Adel MH, Omidvar $\mathrm{B}$, et al. The prevalence and prognostic role of vitamin D deficiency in patients with acute coronary syndrome: a single centre study in south-west of Iran. Heart Lung Circ. 2013;22(5):346-51.

11. Ng LL, Sandhu JK, Squire IB, Davies JE, Jones DJ. Vitamin D and prognosis in acute myocardial infarction. Int J Cardiol. 2013;168(3):2341-6.

12. Belen E, Tipi FF, Aykan AC, Findikçioğlu U, Karakuş G, Yeşil A, et al. Clinical staging in chronic heart failure associated with low vitamin D and elevated parathormone levels. Acta Cardiol. 2014;69(6):665-71.

13. Steg PG, James SK, Atar D, Badano LP, Blömstrom-Lundqvist C, Borger MA, et al. ESC Guidelines for the management of acute myocardial infarction in patients presenting with ST-segment elevation. Eur Heart J. 2012;33(20):2569-619.

14. Hamm CW, Bassand JP, Agewall S, Bax J, Boersma E, Bueno H, et al. ESC Guidelines for the management of acute coronary syndromes in patients presenting without persistent ST-segment elevation: the Task Force for the management of acute coronary syndromes (ACS) in patients presenting without persistent ST-segment elevation of the European Society of Cardiology (ESC). Eur Heart J. 2011;32(23):2999-3054.

15. Kim DH, Sabour S, Sagar UN, Adams S, Whellan DJ. Prevalence of hypovitaminosis D in cardiovascular diseases (from the National Health and Nutrition Examination Survey 2001 to 2004). Am J Cardiol. 2008;102(11):1540-4.

16. Lee JH, Gadi R, Spertus JA, Tang F, O'Keefe JH. Prevalence of vitamin $\mathrm{D}$ deficiency in patients with acute myocardial infarction. Am J Cardiol. 2011;107(11):1636-8.

17. Rodriguez G, Starr AZ, Czernuszewicz GZ, Manhas A, Alhariri A, Willerson JT, et al. Determinants of plasma vitamin D levels in patients with acute coronary syndromes. Eur J Clin Invest. 2011;41(12):1299-309.

18. Matsuoka LY, Wortsman J, Dannenberg MJ, Hollis BW, Lu Z, Holick MF. Clothing prevents ultraviolet-B radiation-dependent photosynthesis of vitamin D3. J Clin Endocrinol Metab. 1992;75(4):1099-103.

19. Correia LC, Sodré F, Garcia G, Sabino M, Brito M, Kalil F, et al. Relation of severe deficiency of vitamin $\mathrm{D}$ to cardiovascular mortality during acute coronary syndromes. Am J Cardiol. 2013;111(3):324-7.

20. Verdoia M, Schaffer A, Sartori C, Barbieri L, Casseti E, Marino P, et al. Vitamin D deficiency is independently associated with extent of coronary artery disease. Eur J Clin Invest. 2014;44(7):634-42.

21. Pittas AG, Chung M, Trikalinos T, Mitri J, Brendel M, Patel K, et al. Systematic review: vitamin D and cardiometabolic outcomes. Ann Intern Med. 2010;152(5):307-14.

22. Alam U, Arul-Devah V, Javed S, Malik RA. Vitamin D and diabetic complications: true or false prophet? Diabetes Ther. 2016;7(1):11-26. 
23. Christodoulidis G, Vittorio TJ, Fudim M, Lerakis S, Kosmas CE. Inflammation in coronary artery disease. Cardiol Rev. 2014;22(6):279-88.

24. De Metrio M, Milazzo V, Rubin M, Cabiati A, Moltrasio M, Marana I, et al. Vitamin D plasma levels and in-hospital and 1-year outcomes in acute coronary syndromes: a prospective study. Medicine (Baltimore). 2015;94(19):e857. doi: 10.1097/MD.0000000000000857.
25. Van Orten-Lutein AC, Janse A, Dhonukshe-Rutten RA, Witkamp RF. Vitamin D deficiency as adverse drug reaction: a cross-sectional study in Dutch geriatric outpatients. Eur J Clin Pharmacol. 2016;72(5):605-14.

Received September 30, 2015 Accepted in revised form July 25, 2016 